\title{
Emerging therapies for atrial fibrillation: is the paradigm shifting?
}

\author{
Ann C. Garlitski • N. A. Mark Estes III
}

Received: 23 January 2010 / Accepted: 24 January 2010/Published online: 3 March 2010

(C) Springer Science+Business Media, LLC 2010

In 1962, Thomas Kuhn popularized the term "paradigm shift" to describe a fundamental change in scientific assumptions in his book entitled "The Structure of Scientific Revolutions" [1]. While some would argue that the term has been overused, most would agree that revolutionary moments that fundamentally alter our understanding of a condition merit this designation. Some consider such a "paradigm shift" to have occurred in the field of cardiac electrophysiology in 1998 with Haissaguerre's publication in The New England Journal of Medicine on "Spontaneous Initiation of Atrial Fibrillation by Ectopic Beats Originating in the Pulmonary Veins" [2]. Over a decade later, the medical community has embraced this mechanistic insight that the triggers of atrial fibrillation (AF) are often found in the pulmonary veins. As a result, we have added AF ablation procedures to our therapeutic armamentarium in an effort to cure this arrhythmia. Due to the heterogeneous nature of AF, involving multiple triggers and substrates which range from structurally normal hearts to complex congenital and acquired abnormalities, our mechanistic knowledge remains rudimentary. While we remain in the infancy of our understanding of this extraordinary common, yet challenging, arrhythmia, we are on the threshold of innovative ablation techniques, multiple novel antiarrhythmic agents, and alternatives to vitamin $\mathrm{K}$ antagonists with the emergence of direct thrombin and factor $\mathrm{Xa}$ inhibitors [3-5]. Each has the

A. C. Garlitski $(\bowtie) \cdot$ N. A. M. Estes III

Tufts New England Cardiac Arrhythmia Center,

The CardioVascular Center, Division of Cardiology,

Department of Medicine, Tufts Medical Center,

Tufts University School of Medicine,

860 Washington Street,

Boston, MA 02111, USA

e-mail: agarlitski@tuftsmedicalcenter.org potential to fundamentally alter the clinical strategies traditionally employed for restoration of normal sinus rhythm and prevention of thromboembolic events. In this respect, it is appropriate to evaluate these emerging therapies and their potential impact on therapeutic strategies for AF over the next several years.

While most of the clinical experience with AF ablation has been with thermal lesions from radiofrequency energy, new energy sources are emerging that have the potential to improve outcomes in AF patients. The emergence of cold as a therapeutic modality can be traced to James Arnott, a well known British physician (1797-1883). Only in recent years, however, with the development of an over-the-wire, deflectable, balloon-based catheter design has the potential for the delivery of cryoenergy in the treatment of $\mathrm{AF}$ become a reality. The novelty lays not only in the energy source, cryothermal energy, rather than conventional radiofrequency energy, but also in the approach to lesions sets, single and circumferential, as opposed to traditional point-by-point ablation. A balloon-based delivery platform may provide a more efficient means of achieving pulmonary vein isolation, particularly suited for patients with paroxysmal $\mathrm{AF}$ in whom the goal is to abolish triggers. Thus far, several European centers have demonstrated procedure safety and short-term efficacy, achieving sinus rhythm in $74 \%$ of patients with paroxysmal AF without the use of antiarrhythmic drug therapy [3]. The major complication appears to be phrenic nerve palsy with ablation of the right-sided veins, whereas no significant pulmonary vein stenosis has been noted [3,6]. Further advances in device configurations will likely address the issues of variable anatomy, and hybrid approaches will be necessary to ablate the substrate in patients with persistent AF. Direct clinical comparisons between radiofrequency and cryoablation will expectedly follow. 
We should remain mindful that catheter-based techniques for curing $\mathrm{AF}$ evolved from seminal laboratory and clinical observations of James Cox who first introduced the surgical Cox-Maze procedure in 1987. Over the last 5 years, there has been enormous renewed interest in the surgical treatment of atrial fibrillation. In large part, this has been a result of the introduction of new ablation technology, which has greatly simplified the operative approach and allowed surgeons to develop less invasive surgical techniques and more widely applicable procedures [7].

With the rapid pace of technological advancements in catheter and surgical ablation, we should continue to adhere to the fundamental principal of evidence-based medicine that safety and efficacy of emerging therapeutic options need to be evaluated in appropriately designed clinical trials and monitored in registries. The potential for growth is staggering in surgical- and catheter-based procedures for $\mathrm{AF}$ ablation, given the estimates that more than 15 million people will have AF by 2050 in the USA alone [8]. Two international registries indicate a growth in number and complexity of catheter ablation procedures for $\mathrm{AF}[9,10]$. Data are available from 20,825 procedures over a 4 -year period from 85 international centers [10]. The success rate was defined as freedom from AF, with or without the use of antiarrhythmic drugs, and it was similar in both surveys at approximately $80 \%$, while the overall complication rate was $4.5 \%$. Although these surveys are helpful in estimating the scope of the procedure, the patient population, success, and complication rates, the voluntary nature introduces inherent bias. A more systematic approach is in its initial stages of development in the USA. In an attempt to be more methodical in collecting patient and procedural data, physicians, professional societies, government, and industry have come together to create the Safety of AF Ablation Registry Initiative [11]. If instituted in the same fashion as the NCDR ICD registry, then it may prove to be a very powerful source of information on the safety and efficacy of $\mathrm{AF}$ ablation procedures.

The development of novel therapeutics is not limited to curative procedures for AF. Novel pharmacologic therapies for restoring and maintaining normal sinus rhythm are emerging from clinical investigation. The latest drug to be added to the list of antiarrhythmic agents is dronedarone, which became available for clinical use in the USA in 2009 [5]. Dronedarone, with the removal of iodine moieties and addition of a methylsulfonamide group, is chemically related to amiodarone. It has antiadrenergic properties and blocks multiple potassium (IKr, IKs, IKur, IK1, ITo), sodium, and L-type calcium channels. The evolution of evidence for the use of dronedarone began with DAFNE, which was a dose-finding trial that demonstrated that the use of $800 \mathrm{mg}$ daily delayed time to first $\mathrm{AF}$ recurrence in patients with persistent AF [12]. EURIDIS and ADONIS evaluated 1,237 patients and determined that, at 12 months, $\mathrm{AF}$ and atrial flutter recurrence was significantly decreased in the dronedarone group. Post hoc analysis demonstrated a $27 \%$ reduction of relative risk of hospitalization and death [13]. The most robust data for the use of dronedarone lie in the results of the ATHENA trial which randomized 4,629 patients with paroxysmal or persistent AF or atrial flutter and revealed a significantly reduced risk of hospitalization due to cardiovascular events or death as well as a decrease in the risk of cardiovascular death by $29 \%(p=0.03)$, a prespecified secondary outcome [5]. The primary end point in ATHENA, the composite of all-cause mortality and cardiovascular hospitalizations, is a novel end point that may indicate changing paradigms of clinical trial endpoints for $\mathrm{AF}$.

It is evident that dronedarone, like all pharmacologic agents, has its advantages and limitations and should be tailored to the unique clinical challenges of each patient. On the one hand, dronedarone has been lauded as a safer alternative to amiodarone. To this extent, there has been one trial, DIONYSOS, which directly compares the two drugs [14]. To date, the study is unpublished, but the pharmaceutical company released study results which favor the safety profile of dronedarone. There was a $20 \%$ decrease in prespecified adverse events, notably less bradycardia and less pronounced QT prolongation. In support of the finding that dronedarone has a superior safety profile, one may also reference a meta-analysis, albeit an "indirect" analysis as each study compared the respective drug against placebo, in which the amiodarone odds ratio (OR) for adverse events leading to drug withdrawal was 1.81 (95\% CI $1.33-2.46$; $p<0.001)$. However, there is a sacrifice in terms of efficacy of preventing $\mathrm{AF}$ recurrence; the $\mathrm{OR}$ for $\mathrm{AF}$ in patients with amiodarone was 0.49 (95\% CI $0.37-0.63 ; p<0.0001)$. The apparent decrease in efficacy must be weighed against the decrease in incidence of systemic side effects on an individual basis. On the other hand, dronedarone, unlike amiodarone, should be avoided in patients with NYHA class III or IV congestive heart failure. In ANDROMEDA, a significantly higher mortality rate, primarily due to exacerbation of congestive heart failure, was noted during a median follow-up of 2 months [15].

Vernakalant may emerge from clinical investigation as an "atrial selective agent" with potential clinical utility for conversion of AF to normal sinus rhythm. It is a multiple ion channel blocker with IKur as its primary target. Since IKur is found in higher density in the atria than in the ventricles, it is, by definition, a more atrial-selective drug. The phase 3 trials of the intravenous form summarily show that vernakalant is moderately effective for the termination of AF [16]. In spite of increases in the QT interval and QRS duration, there were no differences in ventricular arrhythmias between patients taking vernakalant versus placebo. 
Based on the encouraging findings of phase 2 trials of the oral form, we await the completion of phase 3 trials.

In the meantime, we may fortuitously discover the arrhythmic application of drugs which have completed safety and efficacy trials and have already been approved for non-arrhythmic indications. For instance, studies of ranolazine which is approved for the treatment of chronic angina hint at the potential for treatment in the use of arrhythmias. In the canine model, ranolazine suppressed excitability and triggered activity in an isolated pulmonary vein sleeve preparation; it terminated acetylcholine- and isoproterenol-induced atrial fibrillation and prevented its reinitiation [17, 18]. Other "upstream" therapies which inhibit atrial fibrosis and remodeling such as angiotensinconverting enzyme inhibitors and statins with pleotropic effects that include decreasing inflammation are being evaluated.

Pharmaceutical investigations have led us not only to new antiarrhythmic possibilities but also to new agents for the prevention of thromboembolism. Warfarin, which is a synthetic derivative of coumarin, has been both a blessing and a curse. As many patients like to point out, it was initially marketed as a pesticide, but, since then, it has been established as an effective agent that decreases the risk of thromboembolism in patients with AF. However, its metabolism is variable, drug interactions are multiple, and it requires burdensome monitoring to maintain a therapeutic INR range. This decade may witness the introduction of superior alternatives. Disappointingly, a promising direct thrombin inhibitor ximelagatran was not approved for clinical use in the USA due to hepatotoxicity. However, another direct thrombin inhibitor dabigatran etexilate may soon be approved for clinical use in the USA. In a recently published phase III study, RE-LY, the safety and efficacy of the drug was evaluated in 18,113 patients with AF. Despite similar rates of major hemorrhage, dabigatran at $150 \mathrm{mg}$ twice daily is associated with lower rates of stroke and systemic embolism than warfarin [19]. Future studies will also involve AZD 0837, a potent, competitive, reversible inhibitor of free and fibrin-bound thrombin. Direct factor $\mathrm{Xa}$ inhibitors such as rivaroxaban, apixaban, betrixaban, YM 150, and DU176b as well as the indirect factor Xa inhibitor, idrabiotaprinux, are in development [4]. These drugs offer the advantage of minimal protein binding and predictable pharmacokinetics which translates into fixed dosing without laboratory monitoring. In the absence of laboratory monitoring, assessment of compliance is more difficult. Without a specific reversing agent, bleeding may be more difficult to treat.

Thus, we are faced with a hat trick of emerging therapies. Innovative technologies for $\mathrm{AF}$ ablation, novel antiarrhythmic medications, and alternatives to vitamin $\mathrm{K}$ antagonists may fundamentally alter the clinical approach to $\mathrm{AF}$ over the next several years. Whether the clinical trials and registries will be sufficiently robust to result in major changes in guidelines for treatment of $\mathrm{AF}$ remains to be determined. However, if evidence-based medicine does bridge the gap between the promise of these therapies and clinical practice, they will truly represent a paradigm shift in $\mathrm{AF}$ management.

\section{References}

1. Kuhn, T. S. (1962). The structure of scientific revolutions. Chicago: University of Chicago Press.

2. Haissaguerre, M., Jais, P., Shah, D. C., Takahashi, A., Hocini, M., Quiniou, G., et al. (1998). Spontaneous initiation of atrial fibrillation by ectopic beats originating in the pulmonary veins. New England Journal of Medicine, 339(10), 659-666.

3. Neumann, T., Vogt, J., Schumacher, B., Dorszewski, A., Kuniss, M., Neuser, H., et al. (2008). Circumferential pulmonary vein isolation with the cryoballoon technique results from a prospective 3-center study. Journal of the American College of Cardiology, 52 (4), 273-278.

4. Sobieraj-Teague, M., O'Donnell, M., \& Eikelboom, J. (2009). New anticoagulants for atrial fibrillation. Seminars in Thrombosis and Hemostasis, 35(5), 515-524.

5. Hohnloser, S. H., Crijns, H. J., van Eickels, M., Gaudin, C., Page, R. L., Torp-Pedersen, C., et al. (2009). Effect of dronedarone on cardiovascular events in atrial fibrillation. New England Journal of Medicine, 360(7), 668-678.

6. Van Belle, Y., Janse, P., Theuns, D., Szili-Torok, T., \& Jordaens, L. (2008). One year follow-up after cryoballoon isolation of the pulmonary veins in patients with paroxysmal atrial fibrillation. Europace, 10(11), 1271-1276.

7. Estes, N. A., 3rd, \& Damiano, R. J., Jr. (2007). Surgery for atrial fibrillation: current state-of-the-art. Journal of Interventional Cardiac Electrophysiology, 20(3), 57.

8. Miyasaka, Y., Barnes, M. E., Gersh, B. J., Cha, S. S., Bailey, K. R., Abhayaratna, W. P., et al. (2006). Secular trends in incidence of atrial fibrillation in Olmsted County, Minnesota, 1980 to 2000, and implications on the projections for future prevalence. Circulation, 114(2), 119-125.

9. Cappato, R., Calkins, H., Chen, S. A., Davies, W., Iesaka, Y., Kalman, J., et al. (2005). Worldwide survey on the methods, efficacy, and safety of catheter ablation for human atrial fibrillation. Circulation, 111(9), 1100-1105.

10. Cappato R, Calkins H, Chen SA, Davies W, Iesaka Y, Kalman J, Kim YH, Klein G, Natale A, Packer D, Skanes A, Ambrogi F, Biganzoli E. (2009). Up-dated worldwide survey on the methods, efficacy and safety of catheter ablation for human atrial fibrillation. Circulation: Arrhythmia and Electrophysiology doi:10.1161/CIRCEP.109.859116.

11. Al-Khatib, S. (2010). Planning the Safety of Atrial Fibrillation Ablation Registry Initiative (SAFARI) as a collaborative pan-stakeholder critical path registry model: a cardiac safety research consortium " Incubator" think tank. American Heart Journal, 159, 17-24. e11.

12. Touboul, P., Brugada, J., Capucci, A., Crijns, H. J., Edvardsson, N., \& Hohnloser, S. H. (2003). Dronedarone for prevention of atrial fibrillation: a dose-ranging study. European Heart Journal, 24(16), 1481-1487.

13. Singh, B. N., Connolly, S. J., Crijns, H. J., Roy, D., Kowey, P. R., Capucci, A., et al. (2007). Dronedarone for maintenance of sinus rhythm in atrial fibrillation or flutter. New England Journal of Medicine, 357(10), 987-999. 
14. Sanofi-aventis. DIONYSOS Study Results Showed the REspective Profiles of Dronedarone and Amiodarone. http://en.sanofi-aventis. com/binaries/20081223_dionysos_fe_en_en_tcm28-23624.pdf. December 23.

15. Kober, L., Torp-Pedersen, C., McMurray, J. J., Gotzsche, O., Levy, S., Crijns, H., et al. (2008). Increased mortality after dronedarone therapy for severe heart failure. New England Journal of Medicine, 358(25), 2678-2687.

16. Roy, D., Pratt, C. M., Torp-Pedersen, C., Wyse, D. G., Toft, E., Juul-Moller, S., et al. (2008). Vernakalant hydrochloride for rapid conversion of atrial fibrillation: a phase 3, randomized, placebocontrolled trial. Circulation, 117(12), 1518-1525.
17. Burashnikov, A., Di Diego, J. M., Zygmunt, A. C., Belardinelli, L., \& Antzelevitch, C. (2007). Atrium-selective sodium channel block as a strategy for suppression of atrial fibrillation: differences in sodium channel inactivation between atria and ventricles and the role of ranolazine. Circulation, 116(13), 1449-1457.

18. Sicouri, S., Glass, A., Belardinelli, L., \& Antzelevitch, C. (2008). Antiarrhythmic effects of ranolazine in canine pulmonary vein sleeve preparations. Heart Rhythm, 5(7), 1019-1026.

19. Connolly, S. J., Ezekowitz, M. D., Yusuf, S., Eikelboom, J., Oldgren, J., Parekh, A., et al. (2009). Dabigatran versus warfarin in patients with atrial fibrillation. New England Journal of Medicine, 361(12), 1139-1151. 